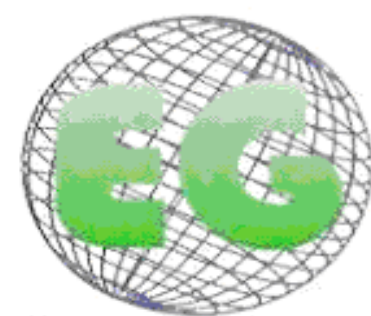

ISSN 1695-6141 $\mathbf{N}^{\circ} 22$
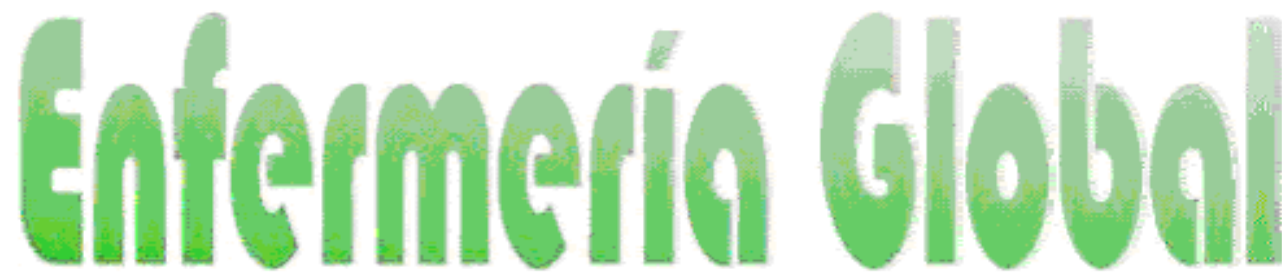

Revista electrónica trimestral de Enfermería

Abril 2011

www.um.es/egloball

CLÍNICA

\title{
ESTUDIO DESCRIPTIVO SOBRE HERIDAS INCISAS POR ACCIDENTE LABORAL
}

\section{DESCRIPTIVE STUDY ABOUT WOUNDS BECAUSE OF WORK ACCIDENT}

\author{
*Reyes Recio, AJ., "Ramírez González, P., **Stagnaro, MA.
}

*DUE de Empresa. Especialista en Enfermería del Trabajo. Técnico Superior en Prevención de Riesgos
Laborales. **Médico Especialista en Urología. Master en Ciencias Médicas (Universidad de Lovaina, Bélgica).

Palabras clave: Herida; Accidente laboral

Keywords: Wounds; Work accident

\section{RESUMEN}

La patología traumática y especialmente las heridas, son uno de los principales motivos de consulta de los usuarios que acuden a nuestros centros asistenciales. El conocimiento de las características, tanto de las heridas como de los trabajadores que las padecen, nos permitirá mejorar la calidad de la asistencia sanitaria prestada y conocer con detalle las duraciones medias de Incapacidad Temporal (IT) para la adecuada y óptima reincorporación del paciente a su puesto de trabajo.

Como objetivos, nos plantemos describir las características de las heridas incisas por accidente laboral, así como también analizar la duración de la IT de las mismas, en los usuarios que acuden a los centros de asistencia primaria de Mutua Universal en la Provincia de Las Palmas.

La investigación que realizamos fue de carácter no experimental, de tipo descriptivo, prospectivo y con diseño transversal. Mediante un cuestionario elaborado específicamente para este estudio, examinamos las características de las heridas incisas de los pacientes que acudieron a nuestros centros de asistencia primaria desde septiembre de 2006 a septiembre de 2007. Tras la recogida de la información se realizó un análisis descriptivo de los datos mediante el paquete estadístico SPSS 12.0.

Como conclusión observamos que la mayoría de los trabajadores que acudieron a nuestros centros de asistencia sanitaria por presentar una herida incisa fue de sexo masculino, diestro, fundamentalmente entre 21 a 40 años de edad, perteneciente al rubro de la construcción, el miembro superior fue el más afectado y las heridas en su gran mayoría tuvieron menos de 6,01 cm de longitud. La cura aplicada más habitual fue mediante sutura y el tratamiento médico coadyuvante analgesia y/o AINE. El proceso cursó sin complicación y precisó, en términos medios, de 5 a 6 días de IT y de 8 a 10 días para la curación. 


\section{ABSTRACT}

Bearing in mind the number of wounds to workers affiliated to Mutual Universal in the whole national territory, we have that $8 \%$ of simple wounds and $4.4 \%$ of complex wounds were attended to in the welfare centers of Mutual Universal in the province of Las Palmas.

In a previous analysis performed at the centers taking part in the study, it was determined that the scrappy wounds were the most frequent, so we focus our study on this type of wounds. From September, 2006 to September, 2007, there was gathered a whole of 289 cases that fulfilled the criteria of incorporation. Knowledge of the characteristics of this type of injuries, always related to the labour activity, will allow us to increase the quality of the sanitary assistance given and to have firsthand knowledge of the average durations of Temporary Invalidity for the suitable and ideal reincorporation of the worker in the work place.

The study that we have carried out has as its principal aim to describe the characteristics of the scrappy wounds for accidents at work of the users who come to the centers of primary assistance of Mutual Universal in the Province of Las Palmas.

\section{INTRODUCCIÓN}

La actividad asistencial que se desarrolla en una Mutua de Accidentes de Trabajo nos obliga a aprovechar al máximo el potencial de recursos humanos y técnicos que tenemos a nuestra disposición para la pronta y satisfactoria recuperación e inserción laboral de nuestros pacientes. En nuestras actuaciones debemos tener siempre presente los ideales de calidad y rapidez en la resolución de los procesos en los que intervenimos, ya que las situaciones de incapacidad temporal conllevan no sólo matices puramente asistenciales sino también económicos y, como entidades colaboradoras de la Seguridad Social, debemos gestionar con eficacia los recursos que utilizamos (1). Durante el desarrollo de nuestra actividad diaria en nuestros centros de trabajo por Contingencias profesionales, la patología que con mayor frecuencia atendemos es la traumática, y dentro de este grupo, destacan las heridas $(2,3)$.

Teniendo en cuenta el número de heridas de los trabajadores afiliados a Mutua Universal a nivel nacional, tenemos que el $8 \%$ de las heridas simples y el $4,4 \%$ de las heridas complejas fueron atendidas en los centros asistenciales de Mutua Universal de la provincia de Las Palmas.

En un análisis previo realizado en los centros participes en el estudio, se determinó que las heridas incisas eran las más frecuentes, por lo que centramos nuestro estudio en este tipo de heridas. Desde septiembre del 2006 a septiembre del 2007, se recogieron un total de 289 casos que cumplieron los criterios de inclusión. El conocimiento de las características de este tipo de lesiones, siempre relacionado con la actividad laboral, nos permitirá mejorar la calidad de la asistencia sanitaria prestada y conocer con detalle las duraciones medias de Incapacidad Temporal (IT) para la adecuada y óptima reincorporación del trabajador a su puesto de trabajo.

El estudio que hemos llevado a cabo, tiene como objetivos describir las características de las heridas incisas por accidente laboral, así como también realizar un análisis de la duración de la IT de las mismas, en los usuarios que acuden a los centros de asistencia primaria de Mutua Universal en la Provincia de Las Palmas. 


\section{MATERIAL Y MÉTODOS}

Se realizó una revisión bibliográfica a través de la Biblioteca Cochrane Plus, base de datos Cuiden ${ }^{\circledR}$ y Google Académico, no encontrando ningún artículo relacionado con heridas incisas en el ámbito laboral, por lo que las comparaciones, en los apartados DISCUSIÓN y CONCLUSIONES, se han realizado con estudios referidos a heridas en general.

Con el fin de conocer qué tipo de heridas eran las más frecuentes, se realizó un análisis previo de los accidentes que acudieron a los centros asistenciales de Mutua Universal en la provincia de Las Palmas durante mayo y junio del 2006 por presentar algún tipo de herida (n $=268$ ). La clasificación de las heridas se hizo teniendo en cuenta el agente físico causante, así tendríamos: excoriaciones, heridas punzantes, heridas incisas, heridas contusas, heridas avulsivas, heridas compuestas y quemaduras (por frío, por calor o químicas). Se observó que las heridas más frecuentes $(31,3 \%)$ eran las de tipo incisas, definidas como aquellas producidas por objetos con bordes cortantes o aristas afiladas (navajas, bisturís, vidrios, etc.), con bordes nítidos, regulares y sin desgarros y localizándose las lesiones específicamente en la zona de dehiscencia o separación tisular (4).

A partir de este dato, iniciamos el estudio propiamente dicho centrándonos en las características de las heridas incisas de los pacientes que acudieron a nuestros centros de asistencia primaria desde septiembre de 2006 a septiembre de 2007.

La investigación fue de carácter no experimental, de tipo descriptivo, prospectivo y con diseño transversal, en el cual se analizaron una serie de casos.

Criterios de inclusión:

- Presentación de una única herida incisa como motivo de consulta.

- El seguimiento desde la recepción del trabajador hasta el momento del alta médica debía ser realizado íntegramente por los servicios sanitarios de Mutua Universal.

Criterios de exclusión:

- Heridas que requirieron tratamiento quirúrgico.

- Pacientes que presentaban otras lesiones, tanto asociadas a la herida (sección tendinosa, lesión vascular....), como no asociadas a la herida (fracturas, contusiones...)

- Pacientes que precisaron la derivación a un centro sanitario ajeno a Mutua Universal durante del tratamiento.

La recopilación de la información se hizo a través de un cuestionario elaborado específicamente para este proyecto (Anexo I), que fue cumplimentado por los DUE's de los diferentes centros asistenciales de Mutua Universal MATEPSS no 10 que participaron en el estudio:

- Centro asistencial de Las Palmas de Gran Canaria (C/ León y Castillo, 222) (Gran Canaria)

- Centro asistencial de Las Palmas de Gran Canaria (C/ Juan Rejón, 67) (Gran Canaria)

- Centro asistencial de Telde (Gran Canaria) 
El tiempo para la recogida de los datos fue de 13 meses (01 de septiembre de 2006 al 28 de septiembre de 2007). Se obtuvieron un total de 289 casos que cumplían los criterios de inclusión.

Las variables analizadas fueron: Edad, Sexo, Zona Corporal, Dedos de la mano lesionados, Mano lesionada, Tamaño, Complicaciones, Sector económico, Profesión, Días de Baja laboral, Días de Curación

Tras la recogida de la información se realizó un análisis descriptivo de los datos mediante el paquete estadístico SPSS 12.0.

\section{RESULTADOS}

Tras el análisis descriptivo de las variables continuas Edad, Días de IT y Días de curación, se obtuvieron los siguientes resultados:

Tabla I. Estadísticas descriptivas + intervalos de Confianza

\begin{tabular}{|l|c|c|c|c|c|c|c|c|c|}
\hline \multicolumn{1}{|c|}{ Tabla I } & $\mathbf{N}$ & Rango & Mínimo & Máximo & Media & IC 95\% & $\begin{array}{c}\text { Error } \\
\text { típico }\end{array}$ & $\begin{array}{c}\text { Desv. } \\
\text { Típica }\end{array}$ & Varianza \\
\hline Edad & $288^{1}$ & 46 & 17 & 63 & $\mathbf{3 5 , 3 1}$ & $\mathbf{3 4 - \mathbf { 3 6 , 3 7 }}$ & 0,602 & 10,213 & 104,299 \\
\hline Días de IT & 289 & 54 & 0 & 54 & $\mathbf{5 , 4 3}$ & $\mathbf{4 , 6 2 - \mathbf { 6 , 2 7 }}$ & 0,418 & 7,109 & 50,533 \\
\hline Días de curación & $252^{2}$ & 53 & 1 & 54 & $\mathbf{9 , 0 9}$ & $\mathbf{8 , 4 1 - 9 , 8 0}$ & 0,354 & 5,613 & 31,506 \\
\hline
\end{tabular}

${ }^{1}$ En la variable Edad hubo 1 caso perdido por déficit en la cumplimentación de la encuesta $(\mathrm{n}=289-1=288)$

${ }^{2}$ En la variable Días de curación hubieron 37 casos perdidos porque el paciente no acudió a las curas sucesivas. $(\mathrm{n}=289-37=252)$

En cuanto a la Edad de los trabajadores lesionados, el $69,5 \%$ de los casos tenía entre 21 y 40 años, siendo la franja etárea más afectada la comprendida entre 31 y 35 años (Gráfico 1) 


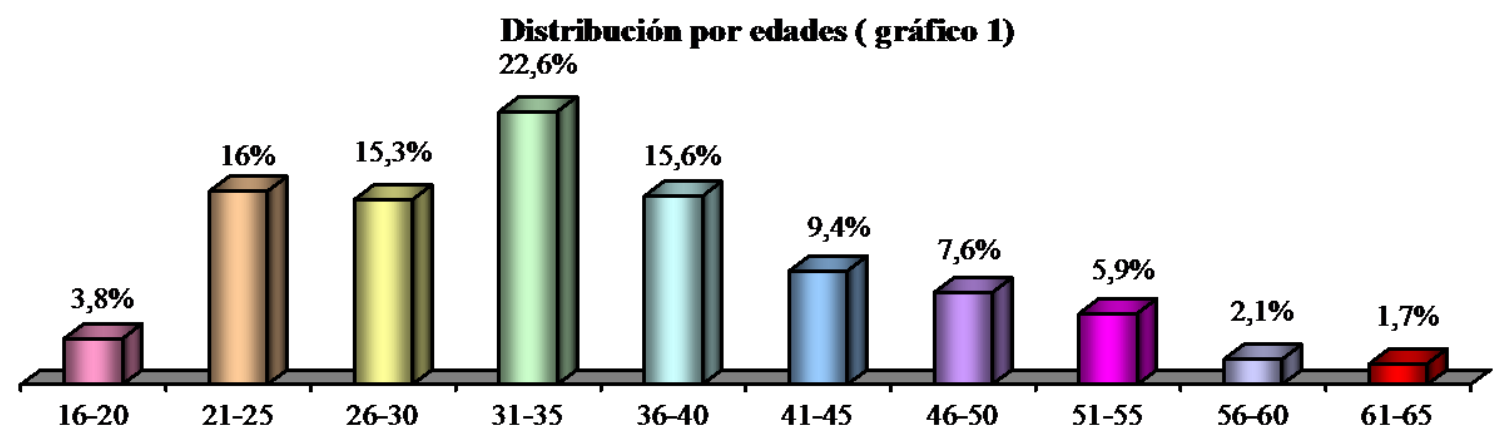

El $74 \%$ ( $n=214)$ de los trabajadores acudió directamente desde el lugar del accidente al centro de asistencia primaria de Mutua Universal, mientras que el $26 \%(n=75)$ lo hizo inicialmente a otro servicio sanitario.

En lo que respecta al Sexo $(n=289)$, el $92,5 \%$ fueron varones y un $7,5 \%$ fueron mujeres.

Del total de trabajadores $(n=289)$ que acudieron con heridas incisas, $162(56,1 \%)$ requirieron baja laboral, mientras que 127 (43,7\%) pudieron continuar con sus actividades laborales habituales. De los trabajadores que requirieron IT $(n=162)$, el $75,7 \%$ permaneció de 1 a 12 días de baja, mientras que el 24,3\% restante necesitó 13 o más días. (Gráfico 2)

\section{Gráfico 2.- Distribución por rangos de días de Invalidez Temporal}

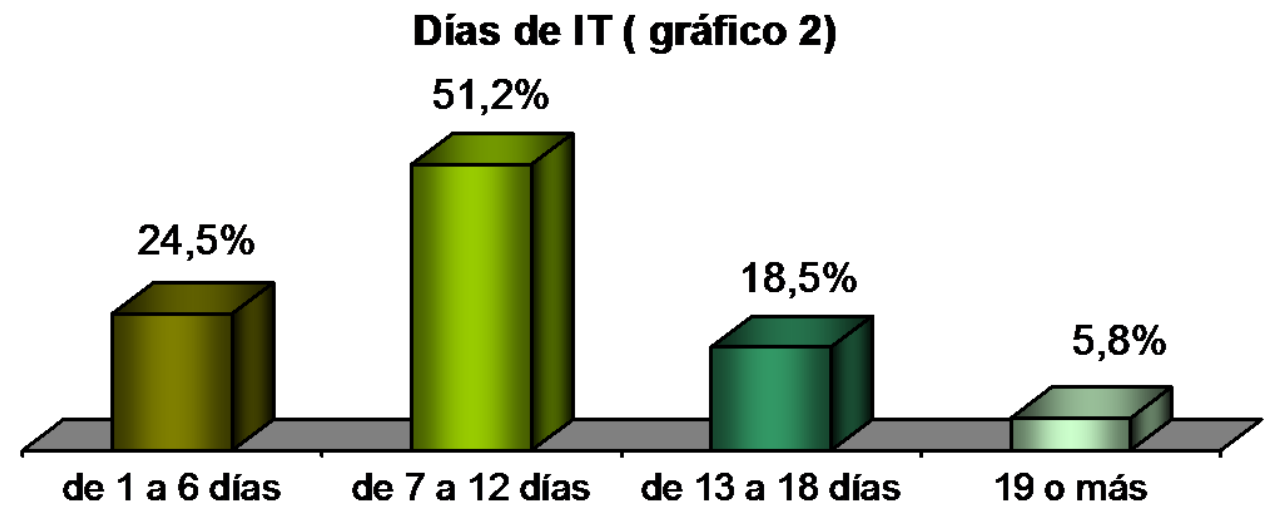

En cuanto al tiempo requerido para la Curación de la herida $(n=252)$, el $83,3 \%$ precisó de 1 a 12 días, mientras que el 16,7\% necesitó 13 o más. (Gráfico 3) 


\section{Días de curación ( gráfico 3)}

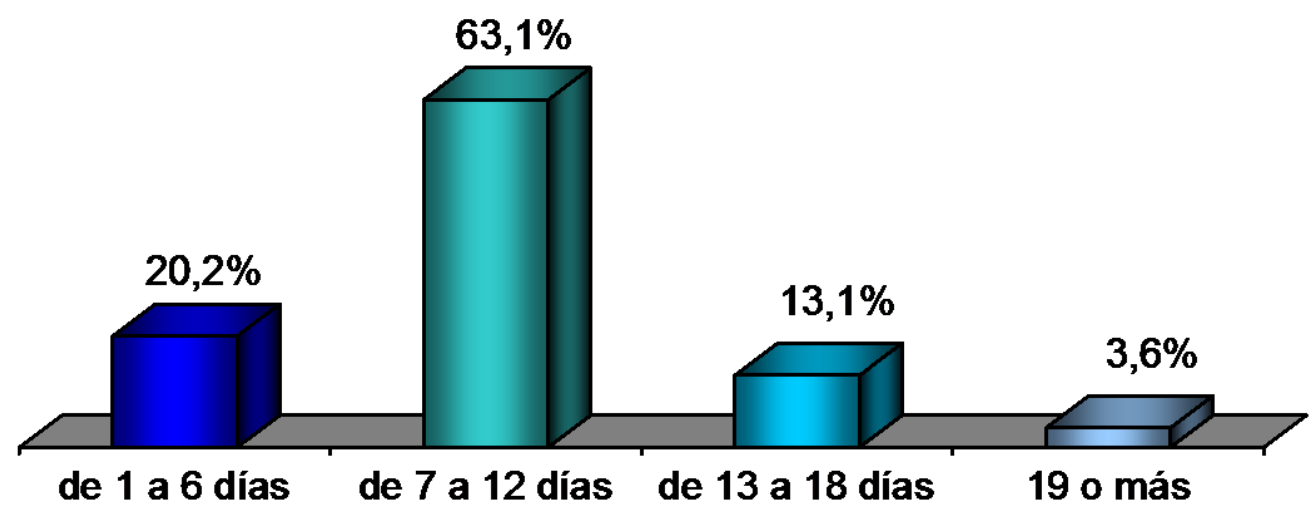

El tipo de Cura aplicada $(n=285)$ más utilizado en las heridas incisas $(86,1 \%)$ ha sido el tratamiento mediante sutura (hilo, grapas y/o sutura cutánea adhesiva), un 4,8\% requirió sutura y cura tópica antibiótica y un $9,1 \%$ sólo requirió tratamiento con cura tópica.

El Tratamiento médico $(\mathrm{n}=289)$ más utilizado fue analgesia y/o AINE asociado a antibiótico $(6,9 \%)$, seguido por analgesia y/o AINE (26\%) y finalmente, sólo antibioticoterapia $(11,1 \%)$. En el $36 \%$ de los casos no se prescribió ningún tratamiento médico coadyuvante.

En cuanto a los Sectores económicos $(\mathrm{n}=289)$, tenemos el mayor número de casos en el sector Servicios (46\%), seguido de la Construcción (34,3\%), la Industria (17\%) y, finalmente, la Agricultura (2,7\%).

Si realizamos un análisis pormenorizado de la Profesión, vemos que la ocupación en la que se producen heridas incisas con mayor frecuencia es en la de peón de la construcción $(25,8 \%)$, seguido por albañil $(21,1 \%)$, conductor en general (grúa, automóvil, camión, guagua, etc) $(6,3 \%)$ y carpintero $(5,9 \%)$.

Considerando la Zona Corporal afectada $(n=289)$, destaca claramente el miembro superior con un $78,2 \%$ de los casos, seguido del miembro inferior $(15,1 \%)$, la cabeza $(5,4 \%)$ y el tronco $(1,3 \%)$. Analizando la ubicación de las heridas con más detalle, vemos que el mayor número de heridas corresponde a los dedos de la mano (46,8\%). Observamos que el $74,4 \%$ del total de heridas se localiza en la franja anatómica que barca desde el antebrazo hasta los dedos de la mano. (Gráfico 4) 


\section{Gráfico 4.- Distribución por Zona Corporal lesionada}

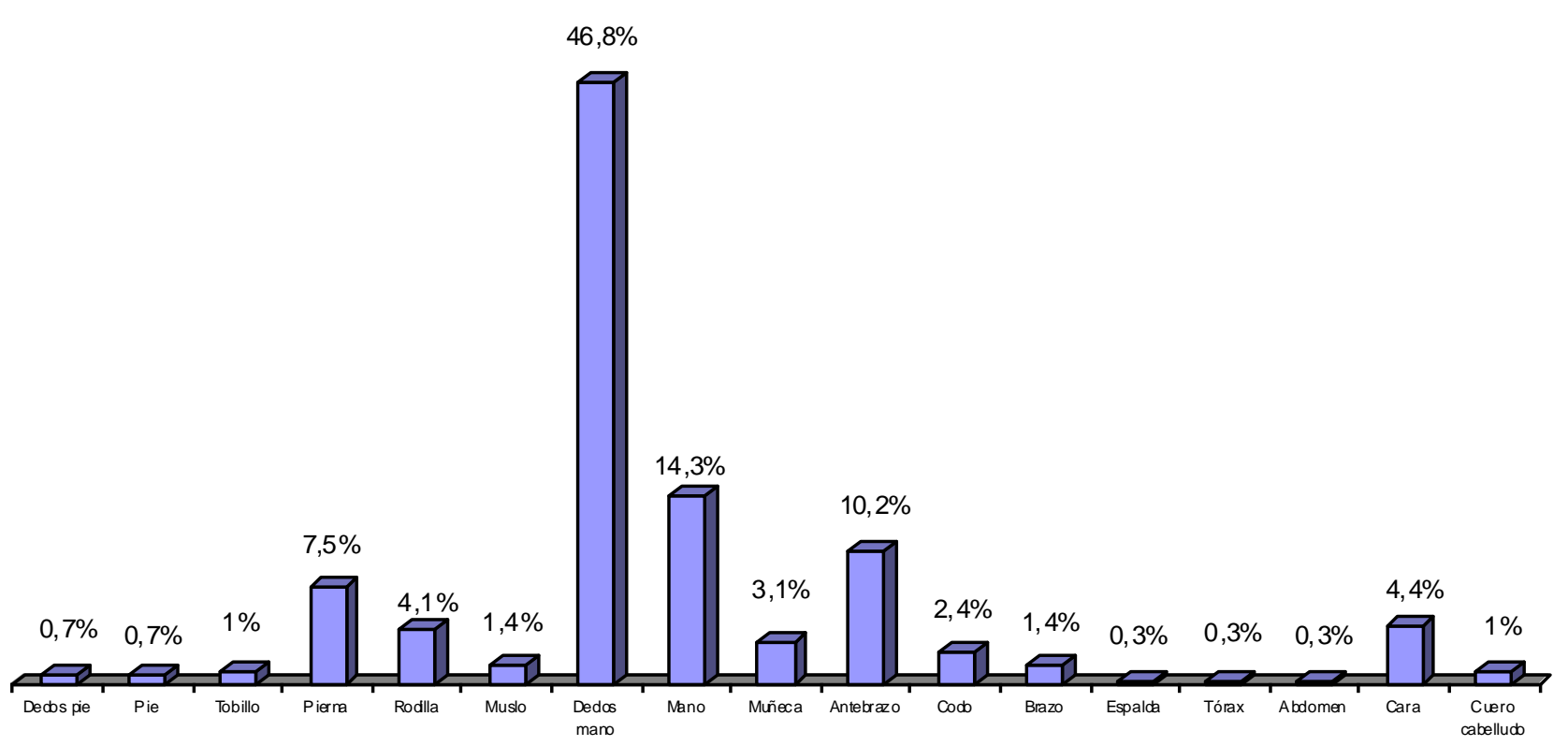

El Dedo afecto de la mano $(n=118)$ que más frecuentemente sufre lesiones $(33,1 \%)$ es el $2^{\circ}$, seguido del $1^{\circ}(28,8 \%)$, el $3^{\circ}$ ( $(17 \%)$, el $5^{\circ}(14,4 \%)$ y el $4^{\circ}(6,7 \%)$.

En relación a la Mano lesionada $(n=186)$, se aprecia una pequeña diferencia entre la derecha $(47,8 \%)$ y la izquierda $(52,2 \%)$.

De los 113 pacientes en los que constaba si su Mano dominante era la derecha o la izquierda, podemos decir que el $87,6 \%$ de los trabajadores eran diestros, mientras que un $12,4 \%$ eran zurdos.

Si consideramos el Tamaño de la lesión $(n=289)$, el $64,5 \%$ de las heridas fueron pequeñas ( $0-3 \mathrm{~cm}$.), el $27,6 \%$ fueron medianas $(3,01-6 \mathrm{~cm}$.) y el $7,9 \%$ fueron grandes (> de $6 \mathrm{~cm}$.).

Un $80,4 \%$ de los casos no presentó Complicaciones durante el tratamiento, mientras que un $11,5 \%$ sufrió dehiscencia, el 2,3\% infección, el 1,9\% necrosis, el 1,2\% maceración, el $1,2 \%$ infección + dehiscencia, el 1,2\% intolerancia a la sutura y, finalmente, un $0,3 \%$ tuvo dehiscencia + necrosis.

En el análisis de la variable Días de IT respecto a la aparición o no de complicaciones, observamos mediante la prueba d de Somers que los días de IT aumentan si aparecen complicaciones durante el proceso de curación $(p=0,001): 4,80$ días de media para las heridas sin complicación frente a 10,98 días de media para las heridas con complicación.

\section{DISCUSIÓN}

Los trabajadores que acudieron con heridas incisas a Mutua Universal se corresponden con una población de Edad joven, con una media de 35,31 años, similar a otros estudios que 
informan de una edad media de 31,5 años (5). Vemos que al igual que en las Estadísticas de accidentes de trabajo del Ministerio de Trabajo y Asuntos Sociales (6) (MTAS), el mayor porcentaje de trabajadores tiene entre 21 y 40 años.

En la variable Sexo, observamos que lo mayoría de trabajadores eran hombres.

La Zona Corporal en la que con mayor frecuencia se producen heridas incisas es en el miembro superior, más concretamente en los dedos de la mano y especialmente en los tres primeros.

En relación a la Mano lesionada, al igual que en el estudio de Tomasina et al (7),se observan más lesiones en la mano izquierda, probablemente porque el mecanismo lesional venga producido al manipular alguna herramienta o máquina cortante con la mano derecha y utilizar la izquierda como apoyo o sujeción del material con que se está trabajando

Observamos un alto porcentaje de casos inferior a 6,01 cm. Este dato puede venir condicionado por los criterios de selección de nuestro trabajo, ya que las heridas de mayor tamaño, habitualmente ocasionan lesiones tendinosas, musculares, vasculares,... quedando fuera de este estudio por no cumplir los criterios de inclusión establecidos.

La Complicación más frecuente fue la dehiscencia de la herida, sin embargo la mayoría de los casos se solucionaron sin agravamiento de la misma.

El Sector económico, con mayor número de casos fue el de Servicios, datos compatibles con las Estadísticas de Accidentes de Trabajo del Ministerio de Trabajo y Asuntos Sociales (6), de las que se extraen las mismas conclusiones.

Si estos datos los desglosamos por Ocupaciones, vemos que al igual que en las Estadísticas de Accidentes de Trabajo del Ministerio de Trabajo y Asuntos Sociales (6) y en el estudio de Martínez et al (5), la ocupación con mayor siniestralidad corresponde a peones de la construcción.

Finalmente, un $43,9 \%$ de los casos precisó Baja laboral, mientras que el $56,1 \%$ de los trabajadores pudo continuar con su labor habitual luego de haber sufrido la lesión. Similares porcentajes se extraen del estudio de Tomasina et al.(7), en el cual el 56\% de los trabajadores no requirió baja laboral, frente al 44\% que si la precisó.

La media de Días de curación de nuestro estudio fue de 9,09 días, sin embargo, los trabajadores pudieron reincorporarse a su puesto de trabajo, luego de una media de 5,43 Días de IT.

\section{CONCLUSIONES}

Tras el estudio que hemos realizado observamos que el perfil de los trabajadores que acudieron a nuestros centros de asistencia sanitaria por presentar una herida incisa fue de un varón, diestro, de 21 a 40 años de edad, de profesión peón de la construcción con una herida incisa en el miembro superior (mayoritariamente el $2^{\circ}$ dedo de la mano izq.) y de tamaño inferior a $6,01 \mathrm{~cm}$. La cura aplicada fue mediante sutura y el tratamiento médico coadyuvante analgesia y/o AINE. El proceso cursó sin complicación y precisó de 5 a 6 días de IT y de 8 a 10 días para la curación ( IC 95\% y con redondeo) 
Destacar que en el $74,4 \%$ de los casos, las heridas incisas se localizaron en la franja anatómica que comprende desde el antebrazo hasta los dedos de la mano. Este dato nos alerta de la importancia de las medidas de protección, tanto individual como colectiva, para dedos, manos y antebrazos, tales como guantes, dispositivos de seguridad en máquinas, etc. que pueden ayudar a disminuir de manera significativa este tipo de accidentes.

En nuestro estudio, puede existir un sesgo de selección que afecte tanto a la validez interna, como a la externa, ya que no se ha utilizado un método riguroso para la selección de la muestra (el muestreo ha sido no aleatorio), por lo que las observaciones e hipótesis que se desprenden del mismo deberán ser contrastadas mediante estudios analíticos 0 experimentales.

\section{BIBLIOGRAFÍA}

1.- M Conde Quintero, MJ Toscano Arroyo, E Estévez Lampón. Tratamiento de heridas con láser infrarrojo. Aproximación a la dosis ideal.. Rev. Cuest. Fisioter. 2009, 38 (1): 25-33

2.- MF Jiménez Navarrete. Accidentes de trabajo y de transito en un área rural de costa rica Rev. Cost. Cienc. Méd. 1989; 10(4);-

3.- Base de datos de Mutua Universal Matepss nํ10 , año 2008.

4.-Ibarra Fernández AJ. Tratado de Enfermería en Cuidados Críticos Pediátricos y Neonatales. [Tratado en Internet]. Madrid; 2006 [acceso 08 de junio de 2009]. Disponible en:

http://www.eccpn.aibarra.org/tratado.htm

5.- Martínez MC, Fragiel J, Rosario de Nava N. Investigación de accidentes laborales con lesiones de las manos y los dedos. Salud de los trabajadores (Venezuela).2003; 11 (2): 99113.

6.- Ministerio de Trabajo y Asuntos Sociales. Estadísticas de accidentes de trabajo [consultado el 26/07/2008] Disponible en:

Http: //www.mtas/estadísticas/EAT/Eat00/Index.htm

7.- Tomasina T, Gómez Echevarne F. Accidentes Laborales en el Hospital de Clínicas. Rev. Med. Uruguay. 2001; 17:156-160 
ANEXO I (CUESTIONARIO DE HERIDAS)

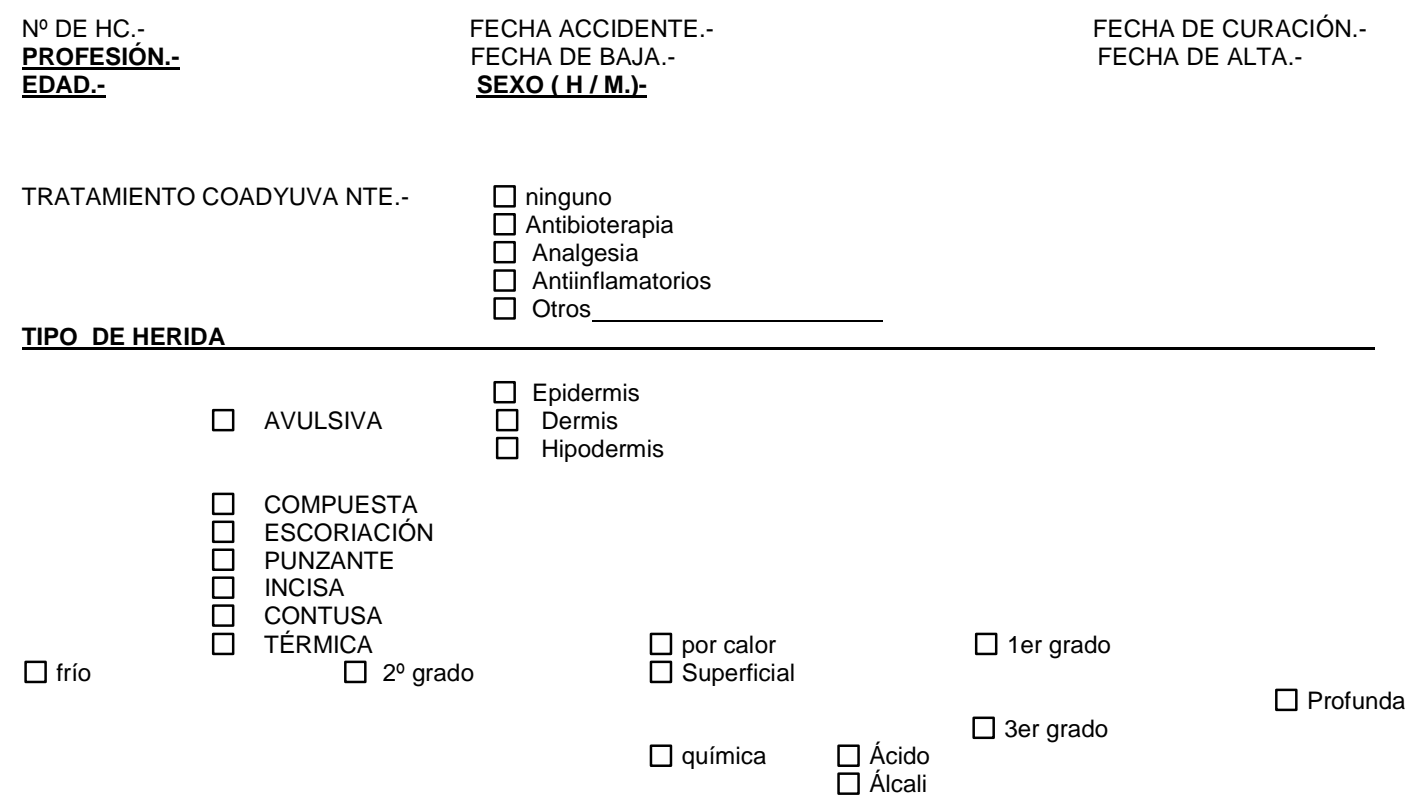

ZONA CORPORAL

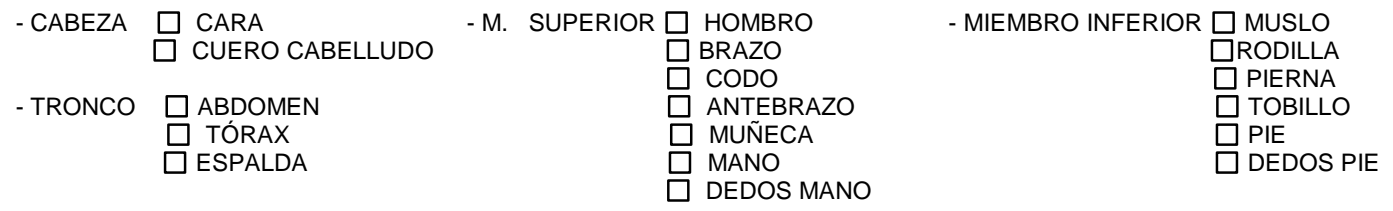

TAMAÑO DE LA HERIDA

$\square$ DE 0 A 3 CM

DE 3,01CM HASTA 6 CM

MÁS DE 6 CM

CURA APLICADA

SUTURA

TIPO DE SUTURA

$\square$ NYLON
$\square$ SEDA
$\square$ OTRAS

TÉCNICA DE SUTURA

SUTURA DOBLE /COLCHONERO

$\square$ TIRAS DE SUTURA CUTÁNEA (steri-strip)

OTRAS

CURA TÓPICA

\section{$\square$ POVIDONA IODADA}

$\square$ NITROFURAZONA (furacin $\odot$ )

SULFADIACINA ARGÉNTICA (silvederma $\odot$ )

$\square$ CLOSTRIDIOPEPTIDASA A (iruxolC)

$\square$ CLOSTRIDIOPEPTIDASA A + neomicina (iruxol neo॰)

$\square$ BETAMETASONA (Celestoderm-V৫)

$\square$ BETAMETASONA + gentamicina (Celestoderm-gentamicina $\odot$ )

$\square$ TRIPSINA, QUIMIOTRIPSONA... (Dertrase $\odot$ )

$\square$ APÓSITO IMPREGNADO (Linitul@)

$\square$ OTROS

\section{COMPLICACIONES}

$\square$ NO $\square$ SÍ $\square$ INFECCIÓN 


\section{$\square$ DEHISCENCIA \\ $\square$ NECROSIS \\ $\square$ OTRAS}

TRATAMIENTO DE LA COMPLICACIÓN

\section{$\square$ POVIDONA IODADA}

$\square$ NITROFURAZONA (furacin $\odot$ )

$\square$ SULFADIACINA ARGÉNTICA (silvederma@)

$\square$ CLOSTRIDIOPEPTIDASA A (iruxol-mono@)

$\square$ CLOSTRIDIOPEPTIDASA A + neomicina (iruxol-neo®)

$\square$ BETAMETASONA (Celestoderm-V())

$\square$ BETAMETASONA + gentamicina (Celestoderm-gentamicina $\odot$ )

$\square$ TRIPSINA, QUIMIOTRIPSONA... (Dertrase $\odot$ )

$\square$ APÓSITO IMPREGNADO (LinitulC)

$\square$ OTROS 